\title{
Maternal mRNA expression levels of H19 are inversely associated with risk of macrosomia
}

Hua Jiang ${ }^{1}$, Yang Yu², Pengcheng Xun ${ }^{3,4}$, Jun Zhang ${ }^{2}$, Guanghua Luo², Qiuwei Wang ${ }^{1}$

\author{
${ }^{1}$ Changzhou Maternity and Child Health Hospital Affiliated to Nanjing Medical \\ University Jiangsu, China \\ ${ }^{2}$ Comprehensive Laboratory, The Third Affiliated Hospital of Soochow University, \\ Changzhou, Jiangsu, China \\ ${ }^{3}$ Department of Nutrition, Gillings Schools of Global Public Health and School \\ of Medicine, University of North Carolina at Chapel Hill, Chapel Hill, USA \\ ${ }^{4}$ Department of Epidemiology, Gillings Schools of Global Public Health, University \\ of North Carolina at Chapel Hill, Chapel Hill, USA
}

Submitted: 7 December 2011

Accepted: 1 April 2012

Arch Med Sci 2014; 10, 3: 525-530

DOI: 10.5114 /aoms.2014.43746

Copyright @ 2014 Termedia \& Banach

\section{Abstract}

Introduction: To investigate the associations between the mRNA levels of H19 in term placenta and risk of macrosomia.

Material and methods: Term placentas were collected from 37 macrosomia and 37 matched neonates with normal birth weight (controls) born in Changzhou Women and Children Health Hospital, Jiangsu province, P. R. China from March 1 to June 30, 2008. The mRNA levels of H19 in those placentas were measured by real-time polymerase chain reaction (PCR). Simple and multiple logistic regression models were used to explore the risk factors in the development of macrosomia. All analyses were performed using Stata 10.0 (StataCorp, College Station, Texas, USA).

Results: The average H19 mRNA level of the macrosomia group was 1.450 \pm 0.456 while in the control group it was $2.080 \pm 1.296$. Based on the result of Student's t test, there was a significant difference in H19 mRNA level between the macrosomia group and the control group $(p=0.008)$. After controlling for potential confounders, the multivariable adjusted odds ratio (OR) of macrosomia for those in the highest tertile of H19 mRNA level was 0.12 (95\% Cl: 0.02-0.59) when compared to those in the lowest tertile ( $p$ for linear trend $=0.009$ ).

Conclusions: The term placental H19 mRNA levels were inversely related to the occurrence of macrosomia. Our findings suggest that the low expression of H19 mRNA may contribute to the development of macrosomia.

Key words: macrosomia, placenta, H19, insulin-like growth factor II, realtime polymerase chain reaction.

\section{Introduction}

Overweight and obesity have increased tremendously during the past $10-15$ years and have become a global health problem. The prevalence of overweight and obesity in Chinese adults has increased two- to three-fold in the past 10-15 years [1,2] and among Chinese children and adolescents it has increased steadily from $5.2 \%$ in 1991 to $13.2 \%$ in 2006 [3]. The prevalence of overweight and obesity increased in all

\author{
Corresponding author: \\ Qiuwei Wang \\ Changzhou Maternity \\ and Child Health Hospital \\ Affiliated to Nanjing Medical \\ University Jiangsu \\ Phone: 086-13915093200 \\ Fax: 086-0519-88109879 \\ E-mail: wangqw126@126.com
}


age groups, in both rural and urban areas, and in North and South China. The overall combined prevalence of overweight and obesity was $23.2 \%$ (17.6\% for overweight and $5.6 \%$ for obesity) in 2002 [4].

Macrosomia has been suggested as one of the possible risk factors for obesity in many studies $[5,6]$. Macrosomia was defined as a condition in which a neonate has a birth weight (BW) equal to or greater than $4000 \mathrm{~g}$. The morbidity of macrosomia reaches $7-10 \%$ [7]. In 2006, the morbidity of macrosomia was $6.5 \%$ in China [8]. Socioeconomic advances and the improvement in women's living standards can predispose to the development of fetal macrosomia, which may result in childhood obesity $[5,6]$. It is a vicious cycle.

The placenta, as the interface between mother and fetus, is central to prenatal growth control. The fetus is dependent upon the placenta for its supply of nutrients and oxygen from the mother. Our previous research found that the placental weights in the macrosomia group were significantly higher than those in the control group and placental weight was positively correlated with birth weight [9]. Recent evidence suggests that imprinted genes may regulate the growth and transport capacity of the placenta by controlling the supply of nutrients [10].

Imprinted genes are expressed in a parent-of-origin manner by epigenetic modifications that silence either the paternal or maternal allele. In general, paternally expressed genes enhance feto-placental growth while maternally expressed genes limit conceptus growth. Insulin-like growth factor II (IGF-II)/H19 is a pair of imprinted genes discovered early. They are similarly positioned, reciprocally imprinted, their expression being correlated with regulation. They co-regulate the growth and transport capacity of the placenta, thereby controlling the supply of nutrients, and are important for fine tuning of normal fetal growth.

Studying the risk factors for macrosomia is of particular public health importance for its primary prevention and therefore reducing the morbidity of obesity.

Previous research focused on whether loss of imprinting status of IGF-II and H19 existed in the placentas of congenital growth disorders such as Beckwith-Wiedemann (BWS) [11], Silver-Russell (SRS) syndromes [12], fetal growth restriction (FGR) [13] and pre-eclampsia [14]. But there has been little research concerning the relation between the expression of IGF-II and H19 and these diseases. However, imprinting status may not be associated with the RNA level [15]. So, it is inconclusive how IGF-II and H19 work: through altering the imprinting status? Changing the RNA level? Or both? Our previous research found that the levels of placental IGF-II were over-expressed in macrosomia [9]. Therefore, in this study we will further investigate the association between the expression of $\mathrm{H} 19$ in the placenta and the occurrence of macrosomia.

\section{Material and methods}

This is a case-control study of the association between mRNA levels of $\mathrm{H} 19$ in the placenta and the occurrence of macrosomia. The study was approved by the human research ethics committees of the Changzhou Women and Children's Health Hospital.

\section{Subjects}

Thirty-seven macrosomia cases were chosen randomly from 116 cases found among children born in Changzhou Women and Children Health Hospital from March 1 to June 30, 2008. Macrosomia was defined as a condition in which neonates had a BW equal to or greater than $4000 \mathrm{~g}$. Thirty-seven neonates with normal BW in the corresponding period were chosen as controls. Mothers of both groups had no diabetes or other complications during pregnancy, confirmed by negative results of a $75 \mathrm{~g}$ oral glucose tolerance test in the $24^{\text {th }}-28^{\text {th }}$ week of pregnancy.

\section{Biomarker detection}

A piece of villus tissue $1 \mathrm{~cm} \times 1 \mathrm{~cm} \times 1 \mathrm{~cm}$ in size was collected from the center of the placenta under aseptic conditions within 5 min after the delivery of the placenta. Then the specimen was cleansed with normal saline (NS) and preserved in liquid nitrogen with Frozen Pipe.

The primers and probes were designed by Primer Premier 5.0 according to the sequence of GAPDH, IGF-II and H19 genes (GenBank accession number NM_002046, J03242 and AK092486, respectively) and synthesized at Shanghai Sangon Biological Engineering Technology and Services Co., LTD. The standard samples of each gene were ordered from Shanghai Shenyou Biological Technology Co., LTD. The sequences of primers and probes are displayed in Table I.

Tissue general RNA was gathered according to steps described in the manual of general RNA extraction kit. $2 \mu \mathrm{g}$ of general RNA was gathered from every tissue. Reverse transcription reaction was done in the qualitative PCR device. All real-time $P C R$ reactions were done on the $A B I 7300$ real-time PCR device. Complex control was done on every specimen. A standard sample was involved in every reaction. The PCR reaction system was composed of: $10 \times$ PBS $2.5 \mu \mathrm{l}, 25 \mathrm{mmol} / \mathrm{l} \mathrm{MgC1}{ }_{2}$ $1.5 \mu \mathrm{l}, 10 \mathrm{mmol} / \mathrm{l} 4 \times \mathrm{dNTPs} 0.5 \mu \mathrm{l}, 100 \mu \mathrm{mol} / \mathrm{l}$ Jus- 
tice primer $0.1 \mu \mathrm{l}, 100 \mu \mathrm{mol} / /$ anti-sense primer $0.1 \mu \mathrm{l}, 100 \mu \mathrm{mol} / /$ probe $0.1 \mu \mathrm{l}, 5 \mathrm{U} / \mu \mathrm{l}$ Taq enzyme $0.25 \mu \mathrm{l}$, specimen $2 \mu \mathrm{l}$. Polymerase chain reaction (PCR) water level was kept at $25 \mu \mathrm{l}$. Reaction conditions went through the following steps: predegeneration at $95^{\circ} \mathrm{C}$ for $1 \mathrm{~min}$, then degeneration at $95^{\circ} \mathrm{C}$ for $5 \mathrm{~s}$, extension of the annealing at $60^{\circ} \mathrm{C}$ for $30 \mathrm{~s}$ (collection of fluorescent signal at this step) and enlarged to 40 cycles. The standard sample was (diluted by 10 times of concentration gradient $\left(10^{8}-10^{3}\right)$ ), and a threshold cycle (CT) value standard curve was drawn on the concentration logs of all standard samples. The CT value was defined as the cycle number when a fluorescent signal was registered in the reaction tube when a set threshold was reached. Then the start copy number was calculated from the standard curve in accordance with the CT value of the unknown specimen.

\section{Covariates}

Information about pregnant women's age, height, education level, weight before pregnancy, weight gain during pregnancy, duration of pregnancy, and time of delivery were collected using an interviewer-administered questionnaire.

\section{Statistical analysis}

Group comparisons of basic characteristics were performed using Student's $t$ test or Wilcoxon's z test as appropriate. Pearson's or Spearman's rank correlation coefficients between indicators were calculated depending on the distributions of the variables. H19 expression levels were divided into three groups according to their tertiles, with the lowest tertile as the reference. Multiple logistic regression models were used to explore the association between $\mathrm{H} 19$ levels and the occurrence of macrosomia.

All analyses were performed using Stata 10.0 (StataCorp, College Station, Texas, USA). Value of $p \leq 0.05$ was considered statistically significant.

\section{Results}

\section{General characteristics of the two groups}

The average BW of the macrosomia group was $4175.8 \pm 178.7 \mathrm{~g}$ while the control group BW was $3230.0 \pm 303.5 \mathrm{~g}$. The macrosomia group had a higher BMI before pregnancy, higher maternal weight before pregnancy and higher weight gain during pregnant. There were no significant differences between cases and controls in maternal age, maternal height, paternal height, paternal weight, paternal BMI, pregnant days or gestational weeks between the two groups (Table II). No significant differences were found in mothers' education level (Wilcoxon's $z=-1.161, p=0.246$ ), family income $(z=0.763, p=0.445)$, or gender of the baby $(z=-0.256, p=0.798)$.

\section{Levels of IGF-II and H19 in the placenta}

The level of IGF-II showed a positively skewed distribution; therefore, median and inter-quartile ranges (Q1-Q3) were used to describe the data. The level of $\mathrm{H} 19$ was normally distributed; therefore, mean and standard deviation were used to describe the data. The levels of IGF-II and H19 expression in placenta of macrosomia and control groups are shown in Table III. Based on the results of Wilcoxon's $z$ test and Student's $t$ test, there were significant differences in IGF-II mRNA and H19 mRNA level between the macrosomia group and the control group.

\section{Correlation between levels of IGF-II and $\mathrm{H} 19$ in the placenta}

Spearman rank correlation analysis suggested that the level of IGF-II had a linear upward trend along with the increase of the $\mathrm{H} 19$ in the normal birth weight group $(r=0.34, p=0.04)$, while

Table I. Sequences of PCR primers and specific probe $\left(5^{\prime}-3^{\prime}\right)$ for the detection of GAPDH, IGF-II and H19 by realtime PCR

\begin{tabular}{|lcc|}
\hline Primer & & Sequence \\
\hline GAPDH & Sense primer & GGA AGG TGA AGG TCG GAG TC \\
\cline { 2 - 3 } & Antisense primer & CGT TCT CAG CCT TGA CGG T \\
\cline { 2 - 3 } & Probe & FAM-TTT GGT CGT ATT GGG CGC CTG-TAMRA \\
\hline IGF-II & Sense primer & CGG CTT CTA CTT CAG CAG GC \\
\cline { 2 - 3 } & Antisense primer & TGG CGG GGG TAG CAC AGT \\
\cline { 2 - 3 } & Probe & FAM-CAA GCC GTG TGA GCC GTC GCA-TAMRA \\
\hline H19 & Sense primer & TCA GGT AGT GCA GTG GTT GTA AAG T \\
\cline { 2 - 3 } & Antisense primer & FAM-AAG CCT GGG CCT TTG AAT CCG GAC A-TAMRA \\
\cline { 2 - 3 } & Probe &
\end{tabular}


Table II. Basic characteristics of pregnant women*

\begin{tabular}{|c|c|c|c|}
\hline Parameter & Macrosomia $^{\dagger}$ & Normal birth weight & Value of $p^{\ddagger}$ \\
\hline No. of participants & 37 & 37 & \\
\hline Maternal age [years] & $27.95 \pm 3.3$ & $27.22 \pm 3.04$ & 0.33 \\
\hline Maternal height & 162.65 & 161.22 & 0.18 \\
\hline Maternal weight before pregnancy & 56.20 & 52.30 & 0.01 \\
\hline BMI before pregnancy & $21.22 \pm 2.36$ & $20.15 \pm 1.58$ & 0.03 \\
\hline Weight gain during pregnancy $[\mathrm{kg}]$ & $20.50 \pm 4.34$ & $17.20 \pm 4.78$ & $<0.01$ \\
\hline Paternal height & 174.08 & 172.89 & 0.28 \\
\hline Paternal weight & 70.62 & 69.97 & 0.79 \\
\hline Paternal BMI & 23.07 & 23.37 & 0.71 \\
\hline Pregnant days & $278.86 \pm 6.36$ & $276.35 \pm 4.79$ & 0.06 \\
\hline
\end{tabular}

${ }^{\star}$ Plus-minus values are mean $\pm S D .{ }^{\dagger}$ Macrosomia is defined as neonates with birth weight $\geq 4000 \mathrm{~g} .{ }^{\ddagger}$ Difference between groups was tested by Student's $t$ test. ${ }^{\S} B M I$ - body mass index, which is the weight in kilograms divided by the square of the height in meters

Table III. Levels of insulin growth factor-II (IGF-II) and H19 in maternal placenta between groups

\begin{tabular}{|lccccc|}
\hline Group & No. of participants & \multicolumn{2}{c|}{ IGF-II mRNA } & \multicolumn{2}{c|}{ H19 mRNA } \\
\cline { 3 - 6 } & & Median & Q1-Q3 & Mean & SD \\
\hline Macrosomia $^{\dagger}$ & 37 & 2.53 & $2.18-4.30$ & 1.45 & 0.46 \\
\hline Normal birth weight & 37 & 1.70 & $0.87-3.55$ & 2.08 & 1.30 \\
\hline Value of $p^{\ddagger}$ & & & $0.03^{*}$ & $<01^{*}$ \\
\hline
\end{tabular}

${ }^{\star} Q 1-Q 3$ denotes inter-quartile range. ${ }^{\dagger}$ Macrosomia is defined as neonates with birth weight $\geq 4000 \mathrm{~g} .{ }^{\ddagger}$ Difference between groups was tested by Wilcoxon's rank sum test and Student's t test

there was no correlation in the macrosomia group $(r=0.19, p=0.25)$.

\section{Association between $\mathrm{H} 19$ levels and risk of macrosomia}

We categorized exposures of interest into tertiles. The initial analysis (model 1) was an unadjusted model. In model 2, we adjusted for maternal height (continuous), maternal weight before pregnancy (continuous) and weight gain during pregnancy (continuous), and in model 3 we additionally adjusted for IGF-II (tertiles). Continuous variables were used for linear trend tests. As compared with those in the lowest tertile of $\mathrm{H} 19$
mRNA level, the multivariable adjusted odds ratio (OR) of macrosomia was 0.22 (95\% Cl: 0.05-0.89) ( $p$ for linear trend $=0.033$ ) for those in the highest tertile (model 2). When further adjusted for IGF-II levels (tertiles), the inverse association was a little strengthened $(\mathrm{OR}=0.12(95 \% \mathrm{Cl}$ : 0.02-0.59); $p$ for linear trend $=0.009$ ) (Table IV).

\section{Discussion}

Our research showed that the level of $\mathrm{H} 19$ in the placenta in macrosomia was significantly lower than that of control group. There was a positive correlation between the levels of IGF-II and H19 in the placenta in normal birth weight. The H19

Table IV. Multivariable-adjusted ORs ( $95 \% \mathrm{Cls})$ of risk of macrosomia by tertiles of $\mathrm{H} 19$ levels

\begin{tabular}{|cccccc|}
\hline H19 mRNA & $\begin{array}{c}\text { Macrosomia } \\
(n=37)\end{array}$ & $\begin{array}{c}\text { Normal BW } \\
(n=37)\end{array}$ & Model I & Model II & Model III $^{\ddagger}$ \\
\hline Tertile 1 & 15 & 10 & 1.00 (Referent) & 1.00 (Referent) & 1.00 (Referent) \\
\hline Tertile 2 & 15 & 10 & $1.00(0.32-3.10)$ & $1.12(0.31-4.02)$ & $1.29(0.31-5.38)$ \\
\hline Tertile 3 & 7 & 17 & $0.27(0.08-0.90)$ & $0.22(0.05-0.89)$ & $0.12(0.02-0.59)$ \\
\hline$P$ for linear trend"l & & 0.033 & 0.033 & 0.009 \\
\hline
\end{tabular}

${ }^{\star}$ All the models were constructed by using logistic regression models. Macrosomia was defined as neonates with birth weight $\geq 4000 \mathrm{~g}$. ${ }^{\dagger}$ Model I: unadjusted model. ${ }^{\ddagger}$ Model II: Adjusted for maternal height (continuous), maternal weight before pregnancy and weight gain during pregnancy (continuous). \$Model III: additionally adjusted for IGF-II (tertiles). "Continuous variables were used for the linear trend tests 
mRNA in the placenta was inversely associated with the occurrence of macrosomia.

\section{The relation between $\mathrm{H} 19$ and IGF-II in placenta and the growth of placenta and macrosomia}

Previous studies on macrosomia mainly discussed gestational nutrition. However, the regulatory function of the placenta is also important. The imprinted genes IGF-II and $\mathrm{H} 19$ are crucial for placental development and fetal growth [10]. The IGF-II gene is paternally expressed and the $\mathrm{H} 19$ gene is maternally expressed. Most of the research paid close attention to whether loss of imprinting of IGF-II and $\mathrm{H} 19$ existed in the placentas of pregnancy-related complications [13-18]. There was loss of imprinting of $\mathrm{H} 19$ in the placentas of fetal growth restriction $[13,17]$ and pre-eclampsia patients $[14,15]$. There was no loss of genomic imprinting of IGF-II and H19 in placentas of diabetic pregnancies with fetal macrosomia [18].

It was suggested that imprinting status did not agree with the RNA level [15]. Therefore, we detected the levels of IGF-II mRNA and H19 mRNA in the placenta to explore whether there was alteration of IGF-II and H19 RNA levels in the macrosomia placenta.

Our research showed that placental expression levels of IGF-II were significantly increased in the macrosomia group $(p=0.025)$ [9], while levels of $\mathrm{H} 19$ were significantly reduced $(p=0.008)$. The over-expression of IGF-II mRNA in the placenta was confirmed as an independent risk factor of macrosomia indicated by both single and multiple logistic regression analysis. The high expression of H19 mRNA in the placenta was confirmed as an independent protective factor of macrosomia (OR $=0.12$, 95\% Cl: 0.02-0.59).

$\mathrm{H} 19$ is a maternally expressed gene that limits conceptus growth. Hence, low expression of H19 may be connected with the placenta in the macrosomia group more than the control group. In a study of intrauterine growth restriction, placental expression levels of $\mathrm{H} 19$ were found to be significantly increased in the FGR group [19]. This is consistent with the hypothesis that low expression of H19 mRNA contributes to macrosomia.

The IGF-II is a paternally expressed gene that enhance feto-placental growth. It was reported that reduced fetal size in IGF-II knockout mice was accompanied by changes in the morphological features and also the size of the placentas in these animals [20,21]. Sibley reported further that IGF-II affected not only the placental development but also the placental transportation ability using a knockout mice experiment [22]. Thus, it is reasonable to speculate that over-expression of IGF-II mRNA contributes to macrosomia.

\section{Factors influencing the level of $\mathrm{H} 19$ in the placenta}

Expression of imprinted genes is classically associated with differential methylation of specific CpG-rich DNA regions (DMR). A methylated DMR on the paternal chromosome inactivates adjacent $\mathrm{H} 19$ and an unmethylated DMR on the maternal chromosome insulates IGF-II from the enhancers.

In a pathophysiological study of preeclamptic, data from methylation-sensitive high resolution melting demonstrated hypermethylation of the promoter region of the $\mathrm{H} 19$ gene, and results of real-time PCR showed decreased mRNA expression of the $\mathrm{H} 19$ gene in early-onset preeclamptic placentas as compared with the normal controls [23].

However, in a pathophysiological study of fetal growth restriction (FGR), placentas from growth-restricted pregnancies had lower methylation levels compared to normals, in the $\mathrm{H} 19$ promoter region. Placental expression levels of $\mathrm{H} 19$ were significantly increased in the FGR group [19]. The hypomethylation of the $\mathrm{H} 19$ promoters is compatible with the aberrant expression.

The over-expression of IGF-II could be caused by deletion or knockout of $\mathrm{H} 19$ [24], while loss of imprinting and hence biallelic expression could downregulate the expression of IGF-II [25]. It is still unclear whether alteration of the $\mathrm{H} 19$ level could regulate directly the expression of IGF-II mRNA and whether the level of H19 mRNA in the macrosomia placenta is involved in regulation of its methylation, which needs to be further investigated.

The mechanisms of these genes' effects on macrosomia will be the focus of our continual study. In view of the factors that may affect the expression of imprinted genes, the methylation of DMR of imprinted genes is most critical. The key of our next study project will be to determine the methylation status of certain CpG islands in the control region on the genomic DNA.

In conclusion, the significant positive associations between the level of placental IGF-II and $\mathrm{H} 19$ and macrosomia were explored. Our findings suggest that a potential risk effect of the value of the expression of IGF-II mRNA and H19 mRNA in the placenta may exist during the development of macrosomia. Further studies are warranted to confirm our findings and clarify the potential mechanisms.

\section{Acknowledgments}

We thank all the study participants for their contributions.

This study was supported by grants from Changzhou Health Bureau (ZD201107) and the Comprehensive Laboratory of the Third Affiliated Hospital of Soochow University. 


\section{References}

1. Wang H, Du S, Zhai F, Popkin BM. Trends in the distribution of body mass index among Chinese adults, aged 20-45 years (1989-2000) Int J Obes (Lond) 2007; 31: 272-8.

2. Wildman RP, Gu D, Muntner P, et al. Trends in overweight and obesity in Chinese adults: between 1991 and 1999. 2000. Obesity (Silver Spring) 2008; 16: 1448-53.

3. Cui Z, Huxley R, Wu Y, Dibley MJ. Temporal trends in overweight and obesity of children and adolescents from nine Provinces in China from 1991-2006. Int J Pediatr Obes 2010; 5: 365-74.

4. Wu YF, Ma GS, Hu YH, et al. The current prevalence status of body overweight and obesity in China: data from the China National Nutrition and Health Survey. Zhonghua Yu Fang Yi Xue Za Zhi 2005; 39: 316-20.

5. Van Eerden P. Obesity in pregnancy. S D Med 2011; Spec No: 46-50.

6. Satpathy HK, Fleming A, Frey D, Barsoom M, Satpathy C, Khandalavala J. Maternal obesity and pregnancy. Postgrad Med 2008; 120: E01-9.

7. Stotland NE, Caughey AB, Breed EM, Escobar GJ. Risk factors and obstetric complications associated with macrosomia. Int J Gynaecol Obstet 2004; 87: 220-6.

8. Yu DM, Zhai FY, Zao LY, et al. Incidence of fetal macrosomia and influencing factors in China in 2006. CJCHC 2008; 16: 11-3.

9. Hua Jiang, Pengcheng Xun, Guanghua Luo, et al. Levels of insulin-like growth factors and their receptors in placenta in relation to macrosomia. Asia Pacif J Clin Nutrition 2009; 18: 171-8.

10. Frost JM, Moore GE. The importance of imprinting in the human placenta. PLoS Genet 2010; 6: 100-15.

11. Cooper WN, Luharia A, Evans GA, et al. Molecular subtypes and phenotypic expression of Beckwith-Wiedemann syndrome. Eur J Hum Genet 2005; 13: 1025-32.

12. Gicquel C, Rossignol S, Cabrol S, et al. Epimutation of the telomeric imprinting center region on chromosome 11 p15 in Silver-Russell syndrome. Nat Genet 2005; 37: 1003-7.

13. Ying W, Jingli F, Wei SW, et al. Genomic imprinting status of IGF-II and H19 in placentas of fetal growth restriction patients. J Genet 2010; 89: 213-6.

14. Zhao D, Li L, Yu LL, et al. H19 expression in placenta with pre-eclampsia. Zhonghua Fu Chan Ke Za Zhi 2009; 44: 87-90.

15. Yu L, Chen M, Zhao D, et al. The H19 gene imprinting in normal pregnancy and pre-eclampsia. Placenta 2009; 30: 443-7.

16. Tycko B. Imprinted genes in placental growth and obstetric disorders. Cytogenet Genome Res 2006; 113: 271-8.

17. Diplas AI, Lambertini L, Lee MJ, et al. Differential expression of imprinted genes in normal and IUGR human placentas. Epigenetics 2009; 4: 235-40.

18. Vambergue A, Fajardy I, Dufour P, et al. No loss of genomic imprinting of IGF-II and H19 in placentas of diabetic pregnancies with fetal macrosomia. Growth Horm IGF Res 2007; 17: 130-6.

19. Koukoura O, Sifakis S, Zaravinos A, et al. Hypomethylation along with increased $\mathrm{H} 19$ expression in placentas from pregnancies complicated with fetal growth restriction. Placenta 2011; 32: 51-7.

20. Reik W, Constancia M, Fowden A, et al. Regulation of supply and demand for maternal nutrients in mammals by imprinted genes. J Physiol 2003; 547: 35-44.
21. Sibley CP, Turner MA, Cetin I, et al. Placental phenotypes of intrauterine growth. Pediatr Res 2005; 58: 827-32.

22. Sibley CP, Coan PM, Ferguson-Smith AC, et al. Placental-specific insulin-like growth factor 2 (igf2) regulates the diffusional exchange characteristics of the mouse placenta. Proc Natl Acad Sci U S A 2004; 101: 8204-8.

23. Gao WL, Li D, Xiao ZX, et al. Detection of global DNA methylation and paternally imprinted $\mathrm{H} 19$ gene methylation in preeclamptic placentas. Hypertens Res 2011; 34: 655-61.

24. Angiolini E, Fowden A, Coan P, et al. Regulation of placental efficiency for nutrient transport by imprinted genes. Placenta 2006; 27 (Suppl A): s98-102.

25. Antonazzo P, Alvino G, Cozzi V, et al. Placental IGF2 expression in normal and intrauterine growth restricted (IUGR) pregnancies. Placenta 2008; 29: 99-101. 